\title{
Malignant melanomas treated by plastic surgeons in Hamilton, Ontario: A retrospective study of 80 patients with clinical and histological correlation
}

\author{
Samih Salama MD FRCPC ${ }^{1}$, Thomas Cherian MD FRCPC ${ }^{1}$, Mitchell Levine MD MSc FRCPC ${ }^{2}$ \\ ${ }^{1}$ Department of Pathology, St Joseph's Hospital, McMaster University, and ${ }^{2}$ Centre for Evaluation of \\ Medicines, St Joseph's Hospital and Department of Clinical Epidemiology and Biostatistics, McMaster \\ University, Hamilton, Ontario
}

\begin{abstract}
S Salama, T Cherian, M Levine. Malignant melanomas treated by plastic surgeons in Hamilton, Ontario: A retrospective study of 80 patients with clinical and histological correlation. Can J Plast Surg 1999;7(4):185-190.

The present retrospective study was designed to analyze the clinical and survival data of 80 patients diagnosed between 1969 and 1987 with confirmed invasive malignant melanoma in Hamilton, Ontario and surrounding region. The age of peak incidence was 31 to 40 years of age for men and 51 to 60 years of age for women. The most frequent type of melanoma was superficial spreading. The most frequent sites were the upper back, ankle and leg. The six-year survival rate for all the patients was $63 \%$. Patients below 50 years of age had a significantly better survival rate than patients above 50 years of age. Survival appears to be inversely proportional to tumour thickness. The findings in this study are similar to those of previous studies regarding clinical features and prognosis. Patient age, and tumour histology and thickness remain important prognostic factors in determining survival for invasive malignant melanoma.
\end{abstract}

Key Words: Canada; Malignant melanoma; Prognosis

Mélanomes malins traités par des plasticiens à Hamilton : une étude rétrospective de 80 patients avec une corrélation clinique et histologique

RÉSUMÉ : La présente étude rétrospective a été conçue pour analyser les données cliniques et de survie de 80 patients diagnostiqués entre 1969 et 1987 avec un mélanome malin invasif confirmé, à Hamilton, en Ontario et dans la région environnante. L'âge de l'incidence maximale se situait entre 31 et 40 ans pour les hommes et de 51 à 60 ans pour les femmes. Le type le plus fréquent de mélanome était celui d'une dissémination superficielle. Les sites les plus fréquents étaient le haut du dos, la cheville et la jambe. Le taux de survie à 6 ans pour tous les patients était de $63 \%$. Les patients de moins de 50 ans démontraient un taux de survie nettement meilleur que ceux âgés de plus de 50 ans. La survie semble inversement proportionnelle à l'épaisseur de la tumeur. Les données recueillies dans cette étude sont similaires à celles des études menées antérieurement pour ce qui est des caractéristiques cliniques et du pronostic. L'âge du patient de même que l'histologie et l'épaisseur de la tumeur demeurent des facteurs pronostiques importants pour déterminer la survie dans le cas d'un mélanome malin invasif.

A ccording to studies performed in different countries, the incidence of malignant melanoma is rising by approximately $3 \%$ to $10 \%$ each year (1-4). In the United States, the number of patients with newly diagnosed melanomas has increased from 14,100 in 1980 to an estimated 25,800 in 1987

Correspondence and reprints: Dr Mitchell Levine, St Joseph's Hospital, 50 Charlton Avenue East, Hamilton, Ontario L8N 4A6. Telephone 905-522-1155 ext 4276, fax 905-521-6136, e-mail levinem@fhs.csu.mcmaster.ca
(5). The incidence of melanoma increased by $83 \%$ even though the United States population increased by only $10.6 \%$ during this seven-year period. Similar increases have also been documented in Canada $(6,7)$. The rapid increase in the incidence of malignant melanoma indicates that it is becoming an increasingly important problem. Increased sun exposure is thought to account largely for this but does not explain the increased incidence in nonsun-exposed skin areas. Melanoma is approximately three to four times more common in lightly pigmented than heavily pigmented races (8). 
TABLE 1

Distribution of patients with malignant melanomas by histological type, age and sex

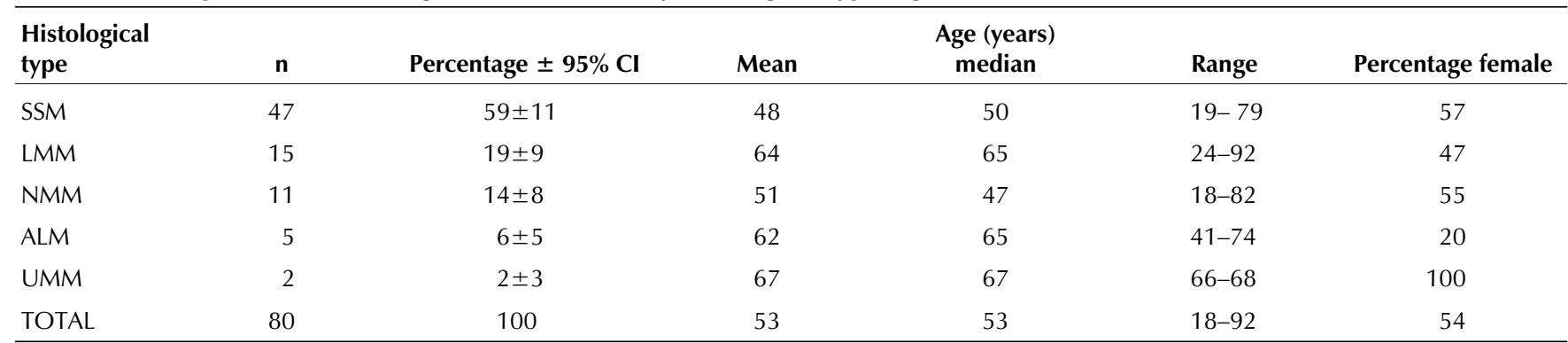

ALM Acral lentiginous melanoma; LMM Lentigo maligna melanoma; NMM Nodular melanoma; SSM Superficial spreading melanoma; UMM Unclassifiable melanoma

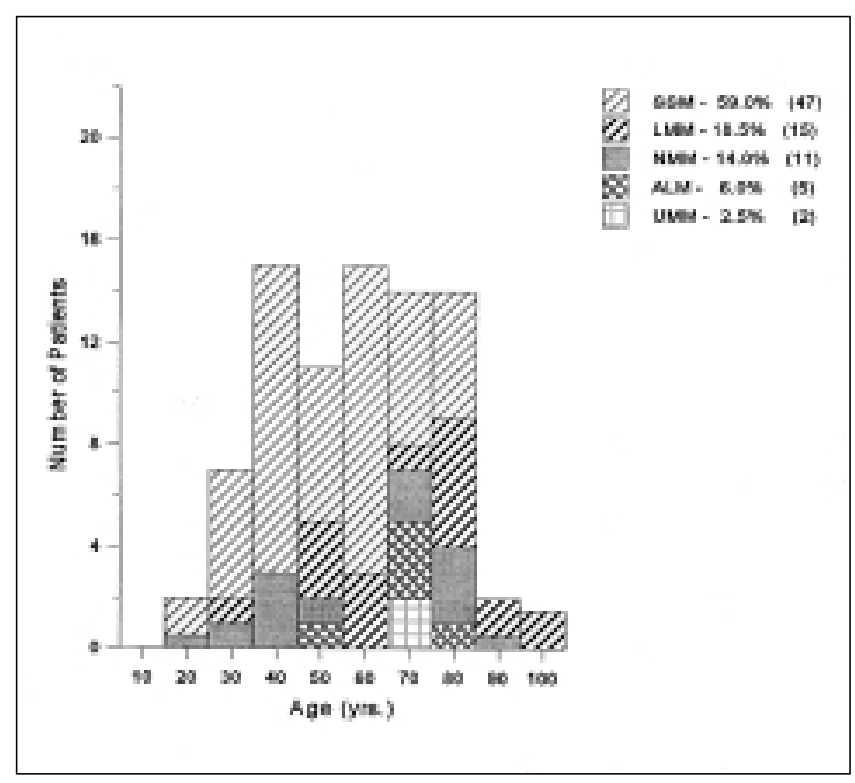

Figure 1) Distribution of 80 patients with malignant melanoma by age and histological type. ALM; Acral lentiginous melanoma; LMM Lentigo maligna melanoma; NMM Nodular melanoma; SSM Superficial spreading melanoma; UMM Unclassifiable melanoma

The increase in mortality rate from melanoma in the United States was relatively uniform at a cumulative rate of about 2.5\%/year from 1950 to 1980 (9). The annual mortality rate from melanoma increased less than $1 \%$ between 1980 and 1983. The five-year survival rate for persons in the United States with melanomas has risen from $41 \%$ in 1945 to $83 \%$ in 1983 (10). Earlier detection and treatment of malignant melanoma have dramatically improved survival in spite of the fact that no new therapies other than surgical treatment have been developed during this interval and that the incidence is rapidly increasing.

In the past 10 years, very few new epidemiological studies of malignant melanoma have been published. However, reviews and analyses of older data sets have continued to demonstrate the importance of tumour thickness (most significant), Clark's level (in skin melanoma) anatomic site and tumour histology (11-14). These results are based on data from the United States and Europe, and do not include Canadian patients. Because of differences in cultures and health care delivery systems between Canada and these countries, we conducted a similar evaluation involving patients treated in Canada. The objectives of the present study were to analyze the material of Canadian patients with malignant melanoma (excluding in situ) in a defined region and to describe the clinical characteristics, histological features, disease management and survival of these patients.

\section{PATIENTS AND METHODS}

From 1969 to 1987,167 consecutive specimens of invasive malignant melanoma were evaluated in Hamilton, Ontario and surrounding areas. The specimens originated from patients referred to plastic surgeons in Hamilton for surgical treatment. The patients' charts and pathology records were reviewed with attention to clinical records and histological findings.

Among these 167 patients, 46 patients were excluded from the study because the original biopsies taken at a referral centre were not available for review, eight because the original biopsies could not be properly assessed, 28 because the lesions were malignant melanoma in situ (level I) and five because no follow-up data were available. Thus, the case histories of a total of 80 patients who had confirmed invasive malignant melanoma with adequate follow-up were studied in the present retrospective review.

Case histories were reviewed for age, sex, site, clinical stage, treatment, disease control and survival. All the patients except one were in clinical stage I at the time of diagnosis. The histological types of melanomas were recorded according to previous studies (ie, superficial spreading melanoma [SSM], lentigo maligna melanoma [LMM], nodular melanoma $[\mathrm{NMM}]$, acral lentiginous melanoma $[\mathrm{ALM}]$ and unclassifiable melanoma [UMM] (15,16). NMMs were diagnosed only when the lesion had a nodular pattern without any adjacent superficial spreading or LMM pattern. The depth of invasion of the tumour was evaluated using Clark's levels (17) and Breslow's measurement of tumour thickness 
TABLE 2

Distribution of patients with malignant melanoma by tumour thickness (Breslow's method)

\begin{tabular}{|c|c|c|c|c|c|c|}
\hline Tumour thickness $(\mathrm{mm})$ & SSM & LMM & NMM & ALM & UMM & Total \\
\hline $0.76-1.50$ & 11 & 6 & 0 & 1 & 0 & 18 \\
\hline$>2.50$ & 9 & 3 & 10 & 0 & 1 & 23 \\
\hline Total & 47 & 15 & 11 & 5 & 2 & 80 \\
\hline
\end{tabular}

ALM Acral lentiginous melanoma; LMM Lentigo maligna melanoma; NMM Nodular melanoma; SSM Superficial spreading melanoma; UMM Unclassifiable melanoma

TABLE 3

Distribution of patients with malignant melanomas by site and histology

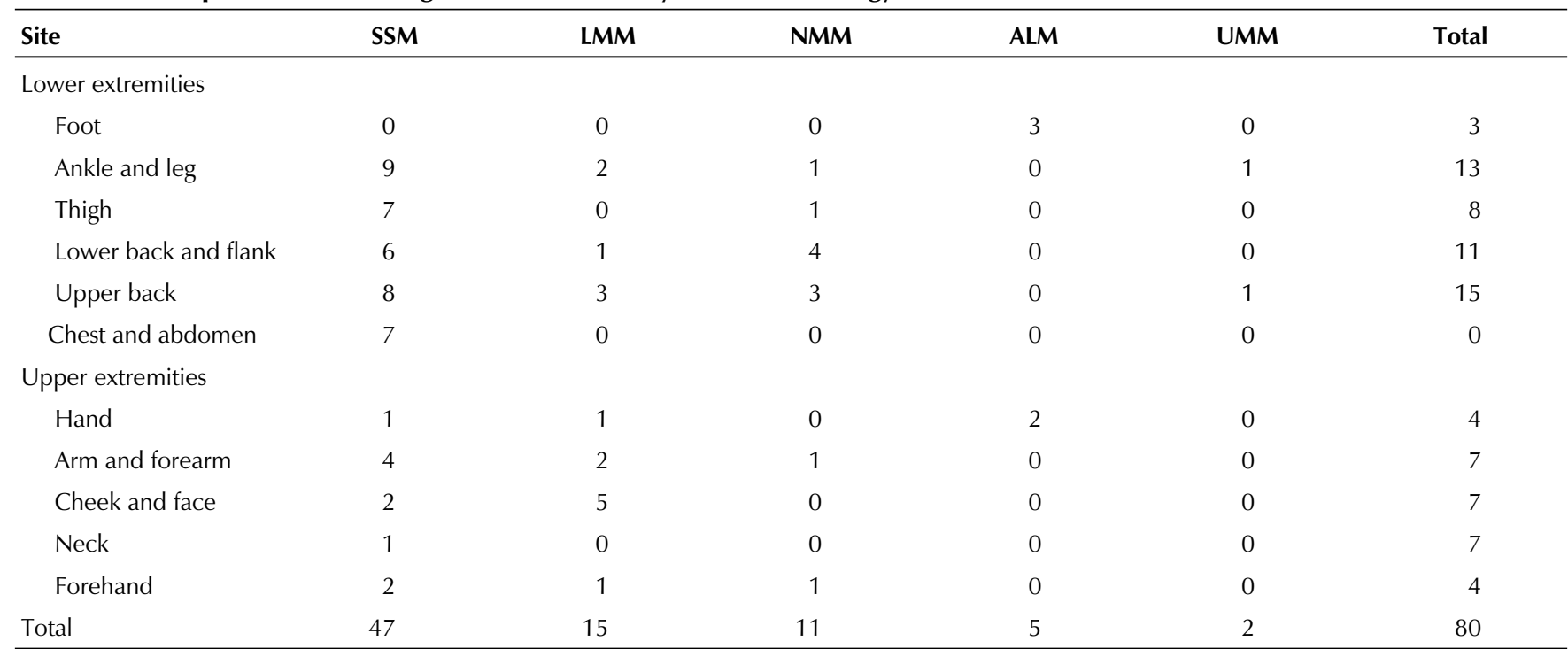

ALM Acral lentiginous melanoma; LMM Lentigo maligna melanoma; NMM Nodular melanoma; SSM Superficial spreading melanoma; UMM Unclassifiable melanoma

(18). The histological slides were reviewed by two pathologists, and the missing data on Clark's levels and tumour thickness were included. Other histological features such as ulceration, vascular invasion, degree of inflammation, regressive changes and amount of melanin were also evaluated.

Survival was calculated by the actuarial method of Cutler and Ederer (19). Survival time was considered as the interval from the date of first histopathological diagnosis to the date of death or the last follow-up. Follow-up consisted of variable intervals, and the last follow-up was obtained in February 1991.

\section{RESULTS}

\section{Age, sex and histology}

The distribution of the melanomas according to age, sex and histology is summarized in Table 1 and Figure 1. The proportions of female and male patients were similar: $54 \%$ female, $\mathrm{P}=0.63$. Peak occurrence was in the 31 to 40 years of age group for males and the 51 to 60 years of age group for females. The median age was 46 years for males and 59 years for females. The median age for the whole group was 53 years. SSM was the most frequent type of melanoma, occurring in 47 patients, and the ALM was the least frequent type. The median ages for SSM and NMM were lower than for the other types, and LMM was associated with an older population.

\section{Levels of invasion and tumour thickness}

The distribution of melanoma patients according to histological type and Clark's levels was assessed. The patients with melanoma in situ (level I) were excluded from the present study. The cases studied included level II invasion - 17 patients $(21 \%)$, level III - $26(32 \%)$, level IV - $29(36 \%)$ and level V $-8(10 \%)$. SMM usually presented at the time of the diagnosis in Level II or III. NMM was usually in level IV or $\mathrm{V}$ at the time of diagnosis, and none were in level II.

The distribution of melanoma cases according to tumour thickness is given in Table 2. The thickness was less than $0.76 \mathrm{~mm}$ in $32 \%$ of the patients, between 0.76 and $1.5 \mathrm{~mm}$ in $22 \%$, between 1.51 and $2.5 \mathrm{~mm}$ in $17 \%$ and greater than $2.5 \mathrm{~mm}$ in $29 \%$ of the patients. The tumour thickness was below $1.5 \mathrm{~mm}$ at the time of diagnosis in $68 \%$ of the SSM cases and $67 \%$ of the LMM cases. All the NMM except one had a thickness greater than $2.5 \mathrm{~mm}$. 


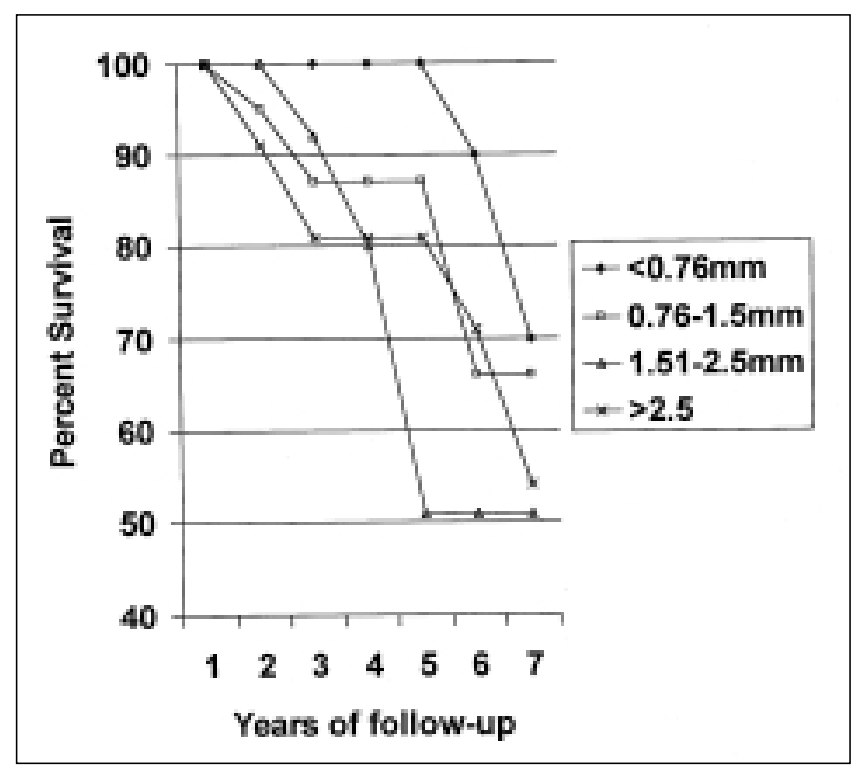

Figure 2) Survival rate according to tumour thickness

\section{Sites}

The distribution of the melanomas according to site and histology is given in Table 3 . The most frequent site was the torso, followed by the lower limbs. All the ALMs, as expected, occurred exclusively in the foot and hand. The NMMs occurred slightly more frequently in the back than in other regions. The face and upper extremities constituted $60 \%$ of the LMM cases. SSMs were more frequent in the trunk and lower extremities.

Ninety-two per cent of ankle and leg melanomas occurred in women; $73 \%$ of upper extremities melanomas were in women, whereas $73 \%$ of melanomas on the upper back of the trunk were in men.

\section{Other histological features}

Ulceration was present in $32 \%$ (26 cases) of the melanomas. Seventy-two per cent of the NMMs and $80 \%$ of the ALMs showed ulceration, whereas only $27 \%$ of the LMMs and $21 \%$ of the SMMs showed ulceration. Ulceration was present in $70 \%$ of the melanomas that had a tumour thickness greater than $2.5 \mathrm{~mm}$.

Vascular invasion was present in seven cases (9\%). Three cases were SSM, two were NMM and two were ALM. All these melanomas except one had either level IV or V invasion. The tumour thickness was greater than $2.5 \mathrm{~mm}$ in five of these cases.

The lymphocytic host response around the melanomas was classified as mild in $27 \%$, moderate in $51 \%$ and severe in $22 \%$. Nine cases $(11 \%)$ had an underlying nevus component.

\section{Survival}

The six-year survival rate for all the patients was $64 \%$. The three-year survival rate was initially better for females, but there was no difference between males and females at six

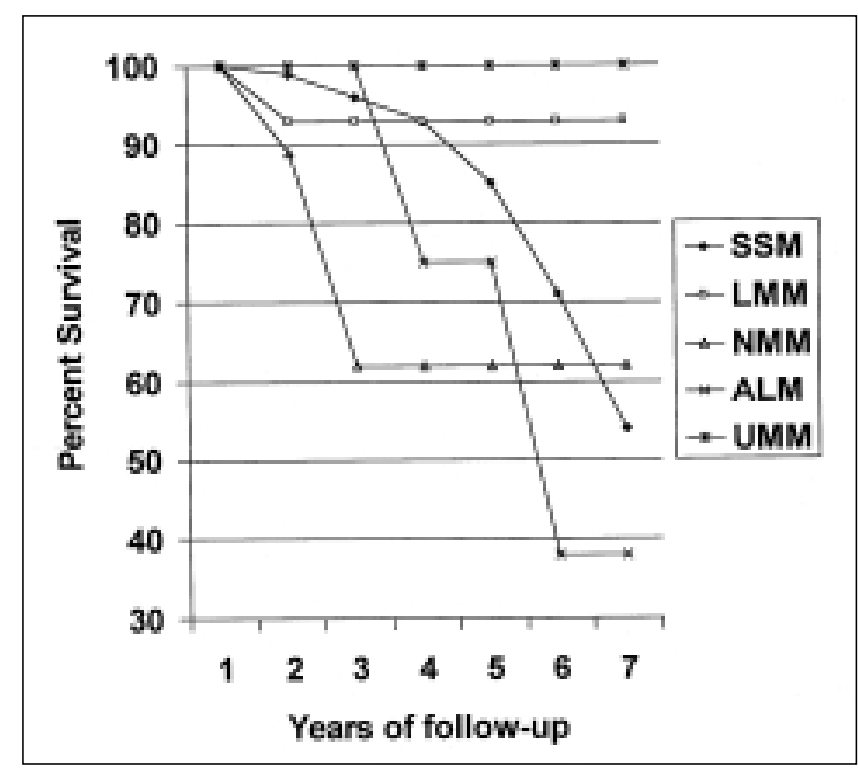

Figure 3) Survival rate according to histological type. ALM Acral lentiginous melanoma; LMM Lentigo maligna melanoma; NMM Nodular melanoma; SSM Superficial spreading melanoma; UMM Unclassifiable melanoma

years. The six-year survival for patients aged 50 years or less was $64 \%$ and for patients more than 50 years of age it was $53 \%(\mathrm{P}<0.01)$.

The 25 patients with tumour thickness of less than $0.76 \mathrm{~mm}$ had a $70 \%$ six-year survival rate. The six-year survival rate for cases with thickness between $0.76 \mathrm{~mm}$ to $1.5 \mathrm{~mm}$ was $66 \%$, for cases between $1.51 \mathrm{~mm}$ to $2.5 \mathrm{~mm}$ it was $50 \%$ and for cases greater than $2.5 \mathrm{~mm}$ it was $56 \%$ (Figure 2). The six-year survival rate for SSM was $54 \%$, for LMM 93\%, for NMM 62\% and for ALM 38\% (P<0.05). Only one of the 15 LMM cases died within six years (Figure 3).

Thirty-three (41\%) of the patients had some form of recurrence or metastases after the initial diagnosis of the melanoma. The 20 patients who died had a mean follow-up of 44 months. The other 13 patients with metastases had a mean follow-up of 40 months. The 47 patients who were diseasefree had a mean follow-up of 40 months.

\section{DISCUSSION}

The present study reaffirms most of the findings of previous studies conducted in different geographic environments (1114,20-23). The age of onset in melanoma patients was most commonly between 30 to 60 years of age, which is in general agreement with the findings of other reports $(20,24)$. The most frequent type of melanoma (SSM) showed a frequency of $59 \%$, which is comparable with that found in other studies $(17,22)$. Some of the studies with large numbers of melanomas showed a high percentage of unclassified melanomas ranging from $15 \%$ to $20 \%$, whereas in our study the percentage of unclassified melanomas was only $2.5 \%$. ALM was also included in this study, which accounted for $6.5 \%$. The proportion of LMMs was slightly higher than in other studies, probably because more cases were diagnosed in sites 
other than the head. Only about $40 \%$ of LMMs occurred in the head. Some studies reported the head as the most frequent site for LMM, ranging from $57 \%$ to $75 \%(17,20,22)$. LMM appears to be a disease of older people, especially when it occurs on the face. LMM at other sites occurred mostly in younger patients.

Most of the previous studies were high-volume studies; hence, they reported more cases at each site. In spite of the relatively small number of cases reviewed in the present study, when categorized into different sites, this study is in agreement with the fact that the most frequent site for malignant melanomas in males is the upper back, and in females it is the lower leg and ankle, even across populations from different geographic areas $(20,25,26,27,28)$.

Fifty-three per cent of patients presented as level II or III at the time of diagnosis. This is comparable with other studies. No nodular melanomas were confined to the papillary dermis level (level II). Most of the NMM were in level IV or $\mathrm{V}$ at the time of diagnosis; only $6 \%$ of other types of melanomas were in level $\mathrm{V}$.

Breslow (18) pointed out that measuring tumour thickness is a more reliable prognostic information than Clark's levels (29). In the present study, the distribution of melanomas according to different tumour thickness are comparable with those of other studies $(18,26)$. All the LMM and most of the SSM cases that had a tumour thickness greater than $2.5 \mathrm{~mm}$ showed a nodular pattern of the invasive tumour. The nodular pattern in these melanomas and in all the NMM resulted in the increased thickness of tumours. There is also a reasonable correlation between the measurement of tumour thickness and Clark's levels. If the tumour thickness is less than $0.76 \mathrm{~mm}$, the tumour is likely to have a level II or level III invasion, and if the thickness is greater than $2.5 \mathrm{~mm}$, it is likely to have level IV or V invasion. The melanomas that have a thickness between these parameters may be of level III or IV.

The five-year survival for melanoma patients has significantly increased during the past 40 years. The five-year survival rate for all the patients in this study was $72 \%$. Male and female survival rates were not significantly different.
Patients less than 50 years of age had a significantly better survival rate than those more than 50 years of age. This is probably due to thinner lesions and earlier diagnosis, and treatment in younger individuals in addition to any differences in other comorbid conditions. In 1969, Clark et al (17) showed that the five-year survival rate decreased with each level, from $92 \%$ in level II to $65 \%$ in level III, $54 \%$ in level IV and $48 \%$ in level V. In this study, data from the next two decades (1970s and 1980s) showed that the patients had a similar prognosis pattern.

Tumour thickness seems to be the most significant prognostic factor. The melanoma cases with less than $0.76 \mathrm{~mm}$ thickness had a $90 \%$ survival, a survival rate similar to that found in other studies $(23,28)$, and survival appears to be inversely proportional to tumour thickness. Local recurrence, and regional and distant metastases correlated very well with the tumour thickness. ALM tumours had the poorest prognosis and the SSM had a variable five-year survival rate, which is similar to what was found in othe studies.

To maximize the internal validity of an epidemiological analysis, the patient population must be from a well defined geographic location. In this study, this was accomplished by using a data set from the Hamilton, Ontario region. The potential issue with this restriction is one of external validity (ie, do the data generalize to populations in other geographical areas of Canada). Although it is not possible to dismiss completely this concern, the general uniformity in standards of health care delivery across the country in the past 25 years makes large regional differences in melanoma prognosis unlikely.

In summary, these data demonstrate the significance of tumour thickness as an important prognostic factor for survival. Clark's levels of invasion can also be used as an adjuvant factor in assessing prognosis. The results of this study demonstrate that melanoma prognostic factors are essentially unchanged over the past two decades (1970s and 1980s). In addition, there appears to be little difference in results from North American, European and Australian/ New Zealand data sources.

\section{REFERENCES}

1. Magnus K. Incidence of malignant melanoma of the skin in the five nordic countries: significance of solar radiation. Int J Cancer 1977;20:477-85.

2. Lee JAH, Peterson GR, Stevens RG, Vesanen K. The influence of age, year of birth and date on mortality from malignant melanoma in the populations of England and Wales, Canada and the white population of the United States. Am J Epidemiol 1979;110:734-79.

3. Holman CDJ, James IR, Gattey PH, Armstrong BK. An analysis of trends in mortality from malignant melanoma of the skin in Australia. Int J Cancer 1980;26:703-9.

4. Anaise D, Steinitz R, Ben Hur N. Solar radiation: a possible etiological factor in malignant melanoma in Israel. Cancer 1978;42:299-304.

5. Rigel DS, Kopf AW, Freidman RJ. The rate of malignant melanoma in the United States: are we making an impact? J Am Acad Dermatol 1987; 17:1050-3.

6. Elwood JM, Lee JAH. Trends in mortality from primary tumours of skin in Canada. Can Med Assoc J 1974;110:913-5.

7. Hu JF. Incidence and mortality of malignant melanoma in Ontario, 1964-1975. Can J Public Health 1985;76:347-51.

8. Crombie IK. Racial differences in melanoma incidence. Br J Cancer 1979;40:185-93.

9. Kopf AW. The rising incidence and mortality rate of malignant melanomas. J Dermatol Surg Oncol 1982;8:760-1.

10. Silverberg E, Lubera J. Cancer statistics. Cancer 1987;37:2-19.

11. Thörn M, Pontén F, Bergström R, Sparén P, Adami HO. Clinical and histopathologic predictors of survival in patients with malignant melanoma: a population-based study in Sweden. J Natl Cancer Inst 1994;86:761-9.

12. Garbe C, Büttner P, Bertz J, et al. Primary cutaneous melanoma. Optimized cutoff points of tumour thickness and importance of Clark's level for prognostic classification. Cancer 1995;75:2484-91. 
13. Berdeaux DH, Meyskens FL Jr, Parks B, et al. Cutaneous malignant melanoma. Cancer 1989;63:1430-6.

14. Morton DL, Davtyan DG, Wanek LA, Foshag LJ, Cochran AJ. Multivariate analysis of the relationship between survival and the microstage of primary melanoma by Clark level and Breslow thickness. Cancer 1993;71:3737-43.

15. McGovern VJ, Mihm MC, Bailly C, et al. The classification of malignant melanoma and its histologic reporting. Cancer 1973;32:1446-57.

16. Lopansri S, Mihm MC Jr. Clinical and pathological correlation of malignant melanoma. J Cutan Pathol 1979;6:180-94.

17. Clark WH Jr, From L, Bernardino EA, Mihm MC. The histiogenesis and biologic behaviour of primary human malignant melanomas of the skin. Cancer Res 1969;29:705-27.

18. Breslow A. Thickness, cross-sectional areas and depth of invasion in the prognosis of cutaneous melanomas. Ann Surg 1970;172:902-8.

19. Cutler S, Ederer F. Maximum utilization of the life table method in analyzing survival. J Chronic Dis 1958;8:699-710.

20. Shaw JHF. Malignant melanoma in Auckland, New Zealand. Surg Gynecol Obstet 1988;166:425-30.

21. Thörn M, Adami HO, Ringborn U, Bergström R, Krusemo UB.
Long-term survival in malignant melanoma with special reference to age and sex as prognostic factors. J Natl Cancer Inst 1987;79:969-74.

22. English DR, Heenan PJ, Holman CDJ, et al. Melanoma in Western Australia in 1980-81: incidence and characteristics of histological types. Pathology 1987;19:383-92.

23. Bonnett A, Roder D, Esterman A. Melanoma case survival rates in South Australia by histological type, thickness and level of tumour at diagnosis. Med J Aust 1986;144:680-2.

24. Cooke KR, Skegg DCG, Fraser J. Trends in malignant melanoma of skin in New Zealand. Int J Cancer 1983;31:715-8.

25. Davis NC, Herron JJ, McLeod GRC. Malignant melanoma of the skin. Lancet 1996;i:91.

26. Balch CM, Murad TM, Soong SJ, et al. tumour thickness as a guide to surgical management of clinical stage I melanoma patients. Cancer 1979;43:883-8.

27. Mackie RM, Hunter JAA. Cutaneous malignant melanoma in Scotland. Cancer 1982;46:75-80.

28. Blois MS, Sagebiel RW, Abarbanel RM, Caldwell TM, Tuttle MS. Malignant melanoma of the skin. Cancer 1983;52:1330-41.

29. Breslow A. tumour thickness, level of invasion and node dissection in stage I cutaneous melanoma. Ann Surg 1975;182:572-5. 